\title{
BICENTRIC QUADRILATERAL CENTRAL CONFIGURATIONS
}

\author{
JAUME LLIBRE ${ }^{1}$ AND PENGFEI YUAN ${ }^{2}$
}

\begin{abstract}
A bicentric quadrilateral is a tangential cyclic quadrilateral. In a tangential quadrilateral the four sides are tangents to an inscribed circle, and in a cyclic quadrilateral the four vertices lie on a circumscribed circle. In this paper we classify all planar central configurations of the 4-body problem, where the four bodies are at the vertices of a bicentric quadrilateral.
\end{abstract}

\section{Introduction AND Statement of the RESUlts}

The well-known Newtonian $n$-body problem concerns with the motion of $n$ mass points with positive mass $m_{i}$ moving under their mutual attraction in $\mathbb{R}^{d}$ in accordance with Newton's law of gravitation.

The equations of the motion of the $n$-body problem are :

$$
\ddot{x}_{i}=-\sum_{j=1, j \neq i}^{n} \frac{m_{j}\left(x_{i}-x_{j}\right)}{r_{i j}^{3}}, \quad 1 \leq i \leq n,
$$

where we have taken the unit of time in such a way that the Newtonian gravitational constant be one, and $x_{i} \in \mathbb{R}^{d}(i=1, \ldots, n)$ denotes the position vector of the $i$-body, $r_{i j}=\left|x_{i}-x_{j}\right|$ is the Euclidean distance between the $i$-body and the $j$-body.

Alternatively the equations of the motion can be written

$$
m_{i} \ddot{x}_{i}=\nabla_{i} U(x), \quad 1 \leq i \leq n,
$$

where $x=\left(x_{1}, \ldots, x_{n}\right)$, and

$$
U(x)=\sum_{1 \leq i<j \leq n} \frac{m_{i} m_{j}}{\left|x_{i}-x_{j}\right|}
$$

is the potential of the system.

\footnotetext{
2010 Mathematics Subject Classification. 70F07,70F15.

Key words and phrases. Convex central configuration, four-body problem, bicentric quadrilateral.
} 
The solutions of the 2-body problem (also called the Kepler problem) has been completely solved. Unfortunately the solutions for the $n$-body $(n \geq 3)$ is still an open problem.

For the Newtonian $n$-body problem the simplest possible motions are such that the configuration is constant up to rotations and scaling. Only some special configurations of particles are allowed in such motions called homographic solutions. Wintner [47] called them central configurations.

More precisely, let

$$
M=m_{1}+\cdots+m_{n}, \quad c=\frac{m_{1} x_{1}+\cdots+m_{n} x_{n}}{M},
$$

be the total mass and the center of masses of the $n$ bodies, respectively.

A configuration $x$ is called a central configuration if the acceleration vectors of the $n$ bodies are proportional to their positions with respect to the center of masses with the same constant of proportionality, i.e.

$$
\sum_{j=1, j \neq i}^{n} \frac{m_{j}\left(x_{j}-x_{i}\right)}{r_{i j}^{3}}=\lambda\left(x_{i}-c\right), \quad 1 \leq i \leq n,
$$

where $\lambda$ is the constant of proportionality.

Equations (1) are invariant under rotations, dilatations and translations on the plane. Two central configurations are related if we can pass from one to the other doing some of the mentioned transformations. This relation is of equivalence. When we talk about the number of central configurations we will talk about the number of classes of equivalence of central configurations.

Central configurations play an important role in Celestial Mechanics, for more details see([18, 23, 34, 36, 42, 45, 47].

There is an extensive literature on the study of central configurations, see Euler [20], Lagrange [27], Albouy and Chenciner [3], Albouy and Fu [4], Albouy and Kaloshin [6], Hampton and Moeckel [25], Llibre [30], Moulton [37] Palmore [38], Schmidt [43], Smale [45], Xia [48, 49], Xie $[50], \ldots$

In this paper we are interested in the planar 4-body problem. For the 4-body problem the exact number and classification of central configurations remain open, only some partial results are obtained. There is a good numerical study on the central configurations of the 4-body prolem, see Simó [44]. The finiteness for the general 4-body problem was 
settled by Hampton and Moeckel using an assisted proof by computer [25], and Albouy and Kaloshin [6] provided an analytical proof.

For $m_{1}=m_{2}=m_{3}=m_{4}$ Llibre found all the planar central configurations assuming the central configurations have an axis of symmetry, see [29]. Later on Albouy proved the existence of such symmetry and provide a more analytical proof.

For the case of three equal masses Bernat at al. classified the noncollinear kite central configurations, see [13], also see [28].

A quadrilateral is convex if none of the vertices is located in the interior of the triangle formed by the other three vertices, otherwise it is concave.

For $m_{1}=m_{2}$ and $m_{3}=m_{4}$, Long and Sun [9] proved some symmetry of the central configurations. Perez-Chavela and Santoprete [40] proved that there is a unique convex non-collinear central configuration of planar 4-body problem when two equal masses are located at opposite vertices of a quadrilateral and, at most, only one of the remaining masses is larger than the equal masses.

When one of the 4 masses is sufficiently small, Pedersen [39], Barros and Leandro $[11,12]$ found the classes of central configurations of the 4-body, see also Gannaway [22] and Arenstorf [10].

For $m_{1}=m_{2} \neq m_{3}=m_{4}$ Álvarez and Llibre [7] characterized the convex and concave central configurations with an axis of symmetry.

Corbera and Llibre [14] gave a complete description of the families of central configurations with two pairs of equal masses when two equal masses are sufficiently small.

Recently Álvarez and Llibre [7] classified Hjelmslev quadrilateral central configurations. A Hjelmslev quadrilateralis a quadrilateral with two right angles at opposite vertices.

A bicentric quadrilateral is a tangential cyclic quadrilateral. In a tangential quadrilateral the four sides are tangents to an inscribed circle, and in a cyclic quadrilateral the four vertices lie on a circumscribed circle, see Figure 1.

A kite quadrilateral is a quadrilateral that two pairs of adjacent sides are of equal length.

In [15] Cors and Robert studied the case when four masses are located at the vertices of a cyclic quadrilateral, see also [9]. 


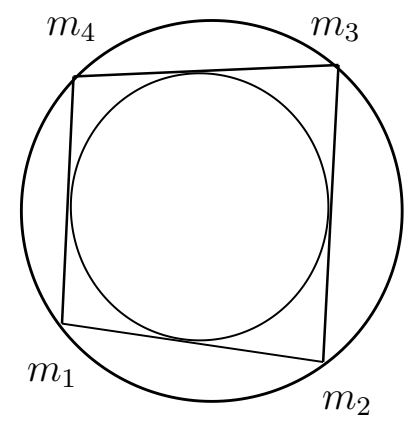

Figure 1. A bicentric quadrilateral.

In this paper we want to classify the bicentric quadrilateral central configurations, i.e. we will improve the classification of the cyclic quadrilateral central configurations provided by Cors and Robert showing which of these central configurations are also tangential quadrilaterals. We characterize all central configurations of the 4-body problem with the four bodies at the vertices of a bicentric quadrilateral.

Theorem 1. Without loss of generality we can take positive masses for the 4-body problem with $m_{1}=1$ and $r_{12}=1$, then

(a) we have a bicentric quadrilateral central configurations with positive masses satisfying $m_{1}=m_{3}=1, \quad r_{12}=r_{23}=1, \quad r_{14}=$ $r_{34}, \quad r_{24}=\sqrt{1+r_{14}^{2}}, \quad r_{13}=2 r_{14} / \sqrt{1+r_{14}^{2}}$, and $r_{14} \in\left(\frac{\sqrt{3}}{3}, 1\right]$,

(b) the shape of a bicentric quadrilateral central configurations is a kite quadrilateral with $r_{14}$ perpendicular to $r_{12}$, and $r_{23}$ perpendicular to $r_{34}$, see Figure 2.

Theorem 1 is proved in Section 3.

We note that from statement $(b)$ of Theorem 1 any bicentric quadrilateral central configurations is a kite Hjelmslev quadrilateral central configuration.

\section{Preliminaries}

Let $x=\left(x_{1}, x_{2}, x_{3}, x_{4}\right) \in\left(\mathbb{R}^{2}\right)^{4}$. We associated with $x$ the matrix:

$$
X=\left(\begin{array}{ccc}
1 & \cdots & 1 \\
x_{1} & \cdots & x_{4} \\
0 & \cdots & 0
\end{array}\right)
$$




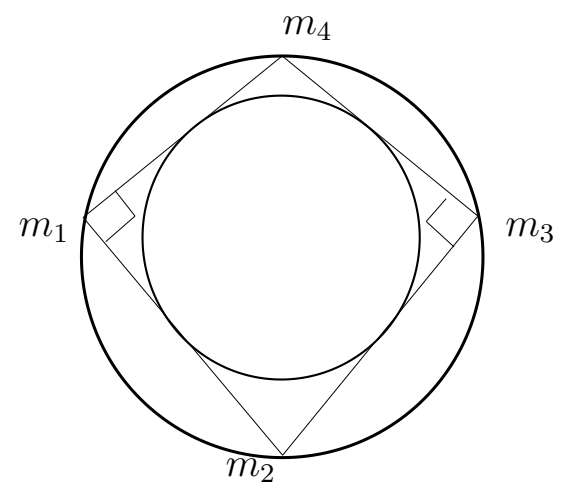

FiguRE 2. The shape of a bicentric quadrilateral central configuration is also a kite Hjelmslev quadrilateral.

$X_{k}$ denotes the matrix obtained deleting from the matrix $X$ its $k$-th column and its last row. Then let $D_{k}=(-1)^{k+1} \operatorname{det}\left(X_{k}\right)$ for $k=$ $1, \ldots, 4$.

$D_{k}$ is twice the signed area of the triangle whose vertices contain all bodies except the $k$-th body, and from the quadrilateral of Figure 1 we have

$$
D_{1}, D_{3}>0, \quad D_{2}, D_{4}<0, \quad D_{1}+D_{2}+D_{3}+D_{4}=0 .
$$

The equations for the central configurations (1) of the 4-body problem were written by Dziobek [16] (see also equations (8) and (16) of Moeckel [34] or [22]) as the following 12 equations with 12 unknowns.

$$
\begin{array}{r}
\frac{1}{r_{i j}^{3}}=c_{1}+c_{2} \frac{D_{i} D_{j}}{m_{i} m_{j}}, \\
t_{i}-t_{j}=0,
\end{array}
$$

for $1 \leq i<j \leq 4$, where

$$
t_{i}=\sum_{j=1, j \neq i}^{4} D_{j} r_{i j}^{2} .
$$

The unknowns of equations (3) are the mutual distances $r_{i j}$, the variables $D_{i}$, and the constants $c_{k}(k=1,2)$.

The first six Dziobek's equation (2) are

$$
\begin{array}{ll}
m_{1} m_{2}\left(r_{12}^{-3}-c_{1}\right)=c_{2} D_{1} D_{2}, & m_{3} m_{4}\left(r_{34}^{-3}-c_{1}\right)=c_{2} D_{3} D_{4}, \\
m_{1} m_{3}\left(r_{13}^{-3}-c_{1}\right)=c_{2} D_{1} D_{3}, & m_{2} m_{4}\left(r_{24}^{-3}-c_{1}\right)=c_{2} D_{2} D_{4}, \\
m_{1} m_{4}\left(r_{14}^{-3}-c_{1}\right)=c_{2} D_{1} D_{4}, & m_{2} m_{3}\left(r_{23}^{-3}-c_{1}\right)=c_{2} D_{2} D_{3} .
\end{array}
$$


Multiplying the two equations which appear in each of the three rows of equations (3) we obtain the same expression $c_{2}^{2} D_{1} D_{2} D_{3} D_{4}$, which provide the Dziobek relation:

$$
\left(r_{12}^{-3}-c_{1}\right)\left(r_{34}^{-3}-c_{1}\right)=\left(r_{13}^{-3}-c_{1}\right)\left(r_{24}^{-3}-c_{1}\right)=\left(r_{14}^{-3}-c_{1}\right)\left(r_{23}^{-3}-c_{1}\right) .
$$

We can solve $c_{1}$ from the Dziobek relation (4) and we have

$$
\begin{aligned}
c_{1} & =\frac{r_{12}^{-3} r_{34}^{-3}-r_{13}^{-3} r_{24}^{-3}}{r_{12}^{-3}+r_{34}^{-3}-r_{13}^{-3}-r_{24}^{-3}} \\
& =\frac{r_{13}^{-3} r_{24}^{-3}-r_{14}^{-3} r_{23}^{-3}}{r_{13}^{-3}+r_{24}^{-3}-r_{14}^{-3}-r_{23}^{-3}} \\
& =\frac{r_{14}^{-3} r_{23}^{-3}-r_{12}^{-3} r_{34}^{-3}}{r_{14}^{-3}+r_{23}^{-3}-r_{12}^{-3}-r_{34}^{-3}} .
\end{aligned}
$$

Defining

$$
\begin{array}{ll}
s_{1}=r_{12}^{-3}+r_{34}^{-3}, & p_{1}=r_{12}^{-3} r_{34}^{-3}, \\
s_{2}=r_{13}^{-3}+r_{24}^{-3}, & p_{2}=r_{13}^{-3} r_{24}^{-3}, \\
s_{3}=r_{14}^{-3}+r_{23}^{-3}, & p_{3}=r_{14}^{-3} r_{23}^{-3},
\end{array}
$$

equation (5) become

$$
c_{1}=\frac{p_{1}-p_{2}}{s_{1}-s_{2}}=\frac{p_{2}-p_{3}}{s_{2}-s_{3}}=\frac{p_{3}-p_{1}}{s_{3}-s_{1}},
$$

which imply that the points $\left(s_{1}, p_{1}\right),\left(s_{2}, p_{2}\right),\left(s_{3}, p_{3}\right)$ of the plane are on the same straight line with slope $c_{1}$, i.e.

$$
\left|\begin{array}{ccc}
1 & 1 & 1 \\
s_{1} & s_{2} & s_{3} \\
p_{1} & p_{2} & p_{3}
\end{array}\right|=0 .
$$

So we can write Dziobek equation as

$D=\left(r_{13}^{3}-r_{12}^{3}\right)\left(r_{23}^{3}-r_{34}^{3}\right)\left(r_{24}^{3}-r_{14}^{3}\right)-\left(r_{12}^{3}-r_{14}^{3}\right)\left(r_{24}^{3}-r_{34}^{3}\right)\left(r_{13}^{3}-r_{23}^{3}\right)=0$.

Lemma 2. If the four masses are at the vertices of a bicentric quadrilateral as in Figure 1, then

$$
\begin{aligned}
& r_{23}=1-r_{14}+r_{34}, \\
& r_{34}=\frac{r_{14}^{2}+r_{13} r_{24}-r_{14}}{1+r_{14}} .
\end{aligned}
$$

Proof. For a bicentric quadrilateral as it is shown in Figure 1 the four sides are tangents to an inscribed circle, and the four vertices lie on a circumscribed circle. 
According to the Pitot theorem [26], we have

$$
r_{12}+r_{34}=r_{23}+r_{14}
$$

and by the Ptolemy's theorem [17], we have

$$
r_{13} r_{24}=r_{12} r_{34}+r_{23} r_{14}
$$

From (9) we can get

$$
r_{23}=r_{12}+r_{34}-r_{14},
$$

then substitute (11) into (10), we obtain

$$
r_{34}=\frac{r_{14}^{2}+r_{13} r_{24}-r_{14}}{1+r_{14}} .
$$

\section{Proof of Theorem 1}

Since we study classes of central configurations, without loss of generality, we can assume $m_{1}=1, r_{12}=1$, and that $r_{12}$ is the longest side of the quadrilateral.

From (3) we can obtain the masses expressed in terms of the distances $r_{i j}$ and the areas $D_{k}$, i.e.

$$
\begin{aligned}
m_{2} & =\frac{D_{2} r_{23}^{3} r_{24}^{3}\left(r_{13}^{3}-r_{14}^{3}\right)}{D_{1} r_{13}^{3} r_{14}^{3}\left(r_{23}^{3}-r_{24}^{3}\right)}, \\
m_{3} & =\frac{D_{3} r_{23}^{3} r_{34}^{3}\left(1-r_{14}^{3}\right)}{D_{1} r_{14}^{3}\left(r_{23}^{3}-r_{34}^{3}\right)}, \\
m_{4} & =\frac{D_{4} r_{24}^{3} r_{34}^{3}\left(1-r_{13}^{3}\right)}{D_{1} r_{13}^{3}\left(r_{24}^{3}-r_{34}^{3}\right)} .
\end{aligned}
$$

Substituting these masses into the first six Dziobek equations (3), and taking only the numerators of these six equations because the denominators do not vanish, we have

$$
\begin{aligned}
e_{1}= & D_{2}\left(c_{2} D_{1}^{3} r_{12}^{3} r_{13}^{3} r_{14}^{2}\left(r_{24}^{3}-r_{23}^{3}\right)-r_{23}^{3} r_{24}^{3}\left(c_{1} r_{12}^{3}-1\right)\left(r_{13}^{3}-r_{14}^{3}\right)\right), \\
e_{2}= & D_{3}\left(c_{2} D_{1}^{2} r_{12}^{3} r_{13}^{3} r_{14}^{3}\left(r_{34}^{3}-r_{23}^{3}\right)-r_{23}^{3} r_{34}^{3}\left(c_{1} r_{13}^{3}-1\right)\left(r_{12}^{3}-r_{14}^{3}\right)\right), \\
e_{3}= & D_{2} D_{3}\left(r_{23}^{3} r_{24}^{3} r_{34}^{3}\left(c_{1} r_{23}^{3}-1\right)\left(r_{12}^{3} r_{14}^{3}\right)\left(r_{14}^{3}-r_{13}^{3}\right)-\right. \\
& \left.c_{2} D_{1}^{2} r_{12}^{3} r_{13}^{3} r_{14}^{6}\left(r_{23}^{3}-r_{24}^{3}\right)\left(r_{23}^{3}-r_{34}^{3}\right)\right) \\
e_{4}= & D_{4}\left(c_{2} D_{1}^{2} r_{12}^{3} r_{13}^{3} r_{14}^{3}\left(r_{34}^{3}\right)-r_{24}^{3}-r_{24}^{3} r_{34}^{3}\left(c_{1} r_{14}^{3}-1\right)\left(r_{12}^{3}-r_{13}^{3}\right)\right),
\end{aligned}
$$




$$
\begin{aligned}
e_{5}= & D_{2} D_{4}\left(-r_{23}^{3} r_{24}^{3} r_{34}^{3}\left(c_{1} r_{24}^{3}-1\right)\left(r_{12}^{3}-r_{13}^{3}\right)\left(r_{13}^{3}-r_{14}^{3}\right)-\right. \\
& \left.c_{2} D_{1}^{2} r_{12}^{3} r_{13}^{6} r_{14}^{3}\left(r_{23}^{3}-r_{24}^{3}\right)\left(r_{24}^{3}-r_{34}^{3}\right)\right) \\
e_{6}= & D_{3} D_{4}\left(c_{2} D_{1}^{2} r_{12}^{6} r_{13}^{3} r_{14}^{3}\left(r_{23}^{3}-r_{34}^{3}\right)\left(r_{34}^{3}-r_{24}^{3}\right)-\right. \\
& \left.r_{23}^{3} r_{24}^{3} r_{34}^{3}\left(c_{1} r_{34}^{3}-1\right)\left(r_{12}^{3}-r_{13}^{3}\right)\left(r_{12}^{3}-r_{14}^{3}\right)\right) .
\end{aligned}
$$

We remark that the last six equations of (2) are identically zero when we substitute the coordinates of the four bodies of Figure 1.

Notice that $D_{i}(i=1,2,3,4)$ is non-zero, so we can eliminate the $D_{i}^{\prime} s$ which appear as a factor in equation (14). First, we solve the first two equations with respect to $c_{1}$ and $c_{2}$, and then we substitute $c_{1}$ and $c_{2}$ in the last four equations of (14). We obtain

$$
\begin{aligned}
e_{3} & =\frac{D}{d} r_{23}^{6} r_{24}^{3} r_{34}^{3}\left(r_{14}^{3}-r_{12}^{3}\right)\left(r_{14}^{3}-r_{13}^{3}\right), \\
e_{4} & =0, \\
e_{5} & =\frac{D}{d} r_{23}^{3} r_{24}^{6} r_{34}^{3}\left(r_{13}^{3}-r_{12}^{3}\right)\left(r_{13}^{3}-r_{14}^{3}\right), \\
e_{6} & =\frac{D}{d} r_{23}^{3} r_{24}^{3} r_{34}^{6}\left(r_{13}^{3}-r_{12}^{3}\right)\left(r_{12}^{3}-r_{14}^{3}\right),
\end{aligned}
$$

where $D=0$ is the Dziobek equation (7), and

$$
d=r_{12}^{3}\left(r_{13}^{3} r_{23}^{3}\left(r_{24}^{3}-r_{34}^{3}\right)+r_{14}^{3} r_{24}^{3}\left(r_{34}^{3}-r_{23}^{3}\right)\right)+r_{13}^{3} r_{14}^{3} r_{34}^{3}\left(r_{23}^{3}-r_{24}^{3}\right),
$$

is the denominator which comes from the denominators of $c_{1}$ and $c_{2}$.

From MacMillan and Bartky [33] we know that for every convex central configuration we have the following inequalities

$$
r_{13}, r_{24}>r_{12}, r_{23}, r_{34}, r_{14} \text {. }
$$

Since $D_{1}, D_{3}>0, D_{2}, D_{4}<0$, and $r_{13}>r_{12}, r_{13}>r_{14}$, the solutions of system (15) are satisfied if and only if $D=0$.

Solving the Dziobeck equation (8), after substituting $r_{12}=1, r_{23}, r_{34}$ from Lemma 1, we get the solution

$$
r_{13}=\frac{2 r_{14}}{r_{24}} .
$$

Then from (8) and (17), for a bicentric quadrilateral central configuration, we have

$$
r_{12}=r_{23}=1, \quad r_{14}=r_{34}, \quad r_{13}=\frac{2 r_{14}}{r_{24}} .
$$

So the bicentric quadrilateral central configurations are also kite quadrilaterals and from (13) we obtain $m_{1}=m_{3}$. 


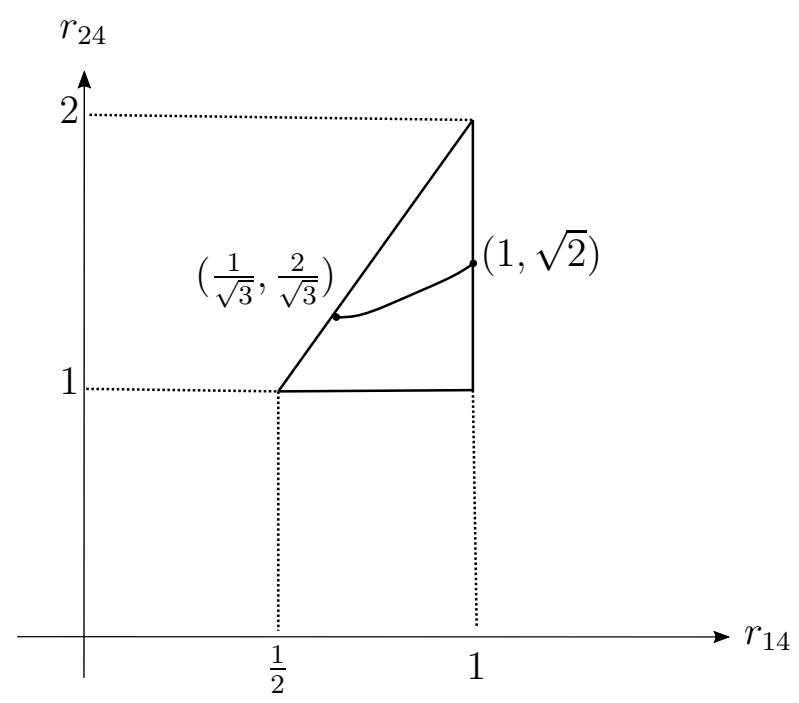

Figure 3.

Since the four bodies are co-circular, and a convex quadrilateral is cyclic if and only if its opposite angles are supplementary [46], we can deduce $r_{14}$ and $r_{12}$ are perpendicular, $r_{23}$ and $r_{34}$ are perpendicular.

Then we have

$$
r_{12}=r_{23}=1, \quad r_{34}=r_{14}, \quad r_{13}=\frac{2 r_{14}}{\sqrt{1+r_{14}^{2}}} \quad r_{24}=\sqrt{1+r_{14}^{2}} .
$$

In order to find the bicentric quadrilateral central configurations with positive masses, from (13), (16), (19) and since $r_{12}=1$ is the longest side of the quadrilateral the following conditions hold

$$
\begin{aligned}
& 1=r_{23}<r_{24}, \\
& r_{34}, r_{14}<r_{13}, r_{24}, \\
& r_{14} \leq 1,1<r_{13}=\frac{2 r_{14}}{r_{24}} .
\end{aligned}
$$

After some simplification, we obtain

$$
r_{14} \leq 1<r_{24}<2 r_{14}<2 .
$$

Denote $\Lambda=\left\{\left(r_{14}, r_{24}\right) \mid r_{14} \leq 1<r_{24}<2 r_{14}<2\right\}$.

By simple computation, the intersection point of $r_{24}=2 r_{14}$ with $r_{24}=\sqrt{1+r_{14}^{2}}$ is $(1 / \sqrt{3}, 2 / \sqrt{3})$, and the intersection point of $r_{14}=$ 1 with $r_{24}=\sqrt{1+r_{14}^{2}}$ is $(1, \sqrt{2})$, then we obtain $r_{14} \in[1 / \sqrt{3}, 1]$. Then for each $r_{14} \in(1 / \sqrt{3}, 1)$ we have a bicentric quadrilateral central configurations with positive masses satisfying (19). 


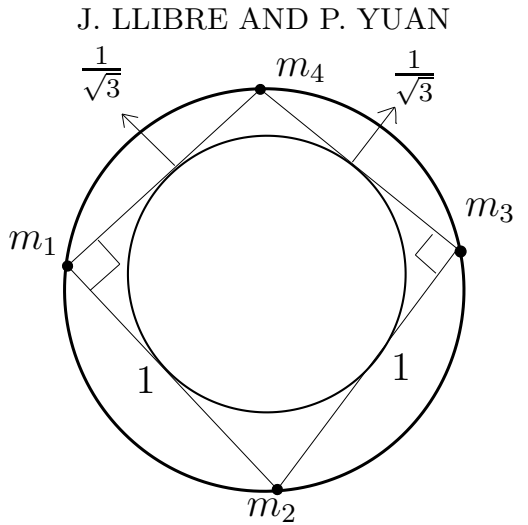

Figure 4.

Now we shall study if we have or not a bicentric quadrilateral central configuration when $r_{14}=1 / \sqrt{3}$ and $r_{14}=1$.

Case for $r_{14}=1 / \sqrt{3}$. From (19), we obtain

$$
r_{12}=r_{23}=1, \quad r_{14}=r_{34}=1 / \sqrt{3}, \quad r_{13}=1, \quad r_{24}=2 / \sqrt{3}
$$

Next we check the masses for this central configuration. From (13) and (21), we have that $m_{2}>0, \quad m_{3}=1, \quad m_{4}=0$, and the central configuration in this case is the kite of Figure 4 but with $m_{4}=0$.

Case for $r_{14}=1$. From (19), we obtain

$$
r_{12}=r_{23}=r_{34}=r_{14}=1, \quad r_{13}=r_{24}=\sqrt{2},
$$

so the configuration is a square. Thus the bicentric quadrilateral central configuration satisfying (22) corresponds to the square central configuration with $m_{1}=m_{2}=m_{3}=m_{4}=1$.

In summary we have bicentric quadrilateral central configurations with positive masses for $r_{i j}$ satisfying

$$
r_{12}=r_{23}=1, \quad r_{34}=r_{14}, \quad r_{24}=\sqrt{1+r+_{14}^{2}}, \quad r_{13}=\frac{2 r_{14}}{\sqrt{1+r+_{14}^{2}}},
$$

where $r_{14} \in\left(1 / \sqrt{3}<r_{14} \leq 1\right]$. This proves the statement $(a)$ of Theorem 1.

\section{ACKNOWLEDGEMENTS}

This work is supported by the Ministerio de Economa, Industria y Competitividad, Agencia Estatal de Investigacin grant MTM201677278-P (FEDER), the Agència de Gestió d'Ajuts Universitaris i de 
Recerca grant 2017 SGR 1617, and the European project DynamicsH2020-MSCA-RISE-2017-777911.

The second author is partially supported by Fundamental Research Funds for the Central Universities (NO.XDJK2015C139), China Scholarship Council(N0.201708505030).

\section{REFERENCES}

[1] Albouy, A., Symétrie des configurations centrales de quatre corps, C. R. Acad. Sci. Paris, 320 (1995), 217-220.

[2] Albouy, A. Recherches sur le problème des n corps, Notes S cientifiques et Techniques du Bureau des Longitudes, Pairs(1997), 78 pp.

[3] Albouy, A., Chenciner, A., Le problème des n corps et les distances mutuelles, Invent. Math. 131 (1998), 151-184.

[4] Albouy, A. and Fu, Y., Euler configurations and quasi polynomial systems, Regul. Chaotic Dyn. 12 (2007), 39-55.

[5] Albouy, A., Fu, Y. and Sun, S., Symmetry of planar four body convex central configurations, Proc. R. Soc. Lond. Ser. A Math. Phys. Eng. Sci., 464 (2008), $1355-1365$.

[6] Albouy, A. and Kaloshin, V., Finiteness of central configurations of five bodies in the plane, Ann. of Math. (2) 176 (2012), 535-588.

[7] Álvarez, M. and Llibre, J., Hjelmslev quadrilateral central configurations, preprint, 2018.

[8] Álvarez, M. and Llibre, J., The symmetric central configurations of the 4body problem with masses $m_{1}=m_{2} \neq m_{3}=m_{4}$, Appl. Math. and Comp. 219 (2013), 599-6001.

[9] Alvarez-Ramirez ,A., Santos, A.A., andVidal, C., On co-circular central configurations in the four and five body-problem for homogeneous force law, J.Dynam. Differential Equations 25 (2013), no.2, 269-290.

[10] Arenstorf, R.F., Central configurations of four bodies with one inferior mass, Cel. Mechanics 28 (1982), 9-15.

[11] Barros, J.F. and Leandro, E.S.G., The set of degenerate cetral configurations in the planar restricted four-body problem, SIAM Journal on Mathematical Analysis 43 (2011), 634-661.

[12] Barros, J.F. and Leandro, E.S.G., Bifurcations and enumeration of classes of relative equilibria in the planar restricted four-body problem, SIAM Joural on Mathematical Analysis 46 (2014), 1185-1203.

[13] Bernat, J., Llibre, J. and Perez Chavela, E., On the planar central configurations of the 4-body problem with three equal masses, Dyn. Contin. Discrete Impuls. Syst. Ser. A Math. Anal. 16 (2009), 1-13.

[14] Corbera, M. and Llibre, J., Central configurations of the 4-body problem with masses $m_{1}=m_{2}>m_{3}=m_{4}=m>0$ and $m$ small, Appl. Math. Comput. 246 (2014), 121-147.

[15] Corbera, M., Cors, J.M.,Llibre, J. and Perez-Chavela, E., Trapezoid central configurations, arXiv.1712.07155[math.DS], 2017.

[16] Cors J.M. and Roberts G.E., Four-body co-circular central configurations, Nonlinearity 25 (2012), 343-370. 
[17] Durell, C.V., Robson,A., Advanced trigonometry, Courier Dover, (2003)[1930].

[18] Dziobek, O., Ueber einen merkwürdigen Fall des Vielkörperproblems, Astro. Nach. 152 (1900), 32-46.

[19] Érdi, B. and Czirják, Z., Central configuration of four bodies with an axis of symmetry, Celestial Mech. Dynam. Astronom. 125 (2016), no.1, 33-70.

[20] Euler, L., De moto rectilineo trium corporum se mutuo attahentium, Novi Comm. Acad. Sci. Imp. Petrop., 11 (1767), 144-151.

[21] Fernandes, A.C., Llibre, J. and Mello, L.F., Convex central configurations of the 4-body problem with two pairs of equal masses, Arch. Rational Mech. Anal. 226 (2017), 303-320.

[22] Gannaway, J.R., Determination of all central configurations in the planar 4body problem with one inferior mass, Ph. D., Vanderbilt University, Nashville, USA, 1981.

[23] Hagihara, Y., Celestial Mechanics, vol. 1, MIT Press, Massachusetts, 1970.

[24] Hartshorne, R., Geometry: Euclid and Beyond, Springer, 2005, pp. 429-430.

[25] Hampton, M. and Moeckel, R., Finiteness of relative equilibria of the four-body problem, Invent. Math. 163 (2006), no.2, 289-312.

[26] Josefson, M., More characterizations of tangential quadrilaterals, Forum Geometricorum, 11, 65-82.

[27] Lagrange, J.L., Essai surle probléme des trois corps, recueil des pièces qui ont remporté le prix de l'Académie royale des Sciences de Paris,tome IX,1772, reprinted in Ouvres, Vol.6 (Gauthier-Villars, Paris, 1873), pp 229-324.

[28] Leandro, E.S.G., Finiteness and bifurcation of some symmetrical classes of central configurations, Arch. Rational Mech. Anal. 167 (2003), 147-177.

[29] Llibre, J., Posiciones de equilibrio relativo del problema de 4 cuerpos, Publica cions Matemàtiques UAB3 (1976), 73-88.

[30] Llibre, J., On the number of central configurations in the N-body problem, Celestial Mech. Dynam. Astronom. 50 (1991), 89-96.

[31] Long, Y., Admissible shapes of 4-body non-collinear relative equilibria, Adv. Nonlinear Stud. 3 (2003), no. 4, 495-509.

[32] Long, Y. and Sun, S., Four-Body Central Configurations with some Equal Masses, Arch. Rational Mech. Anal. 162 (2002), 24-44.

[33] MacMillan, W.D. and Bartky, W., Permanent Configurations in the Problem of Four Bodies, Trans. Amer. Math. Soc. 34 (1932), no. 4, 838-875.

[34] Moeckel, R., On central configurations, Mathematische Zeitschrift 205 (1990), no. 4, 499-517.

[35] Moeckel, R., Generic finiteness for Dziobek configurations, Trans. Amer. Math. Soc. 353 (2001), 4673-4686.

[36] Meyer, K.R., Bifurcation of a central configuration, Cel.Mech. 40 (1987), 273282.

[37] Moulton, F.R., The straight line solutions of n bodies, Ann. of Math. 12 (1910), $1-17$.

[38] Palmore, J.I.,Classifying relative equilibria II, Bull. Amer. Math. Soc. 81(1975), 71-73.

[39] Pedersen, P., Librationspunkte im restringierten Vierk" orperproblem, Danske Vid. Selsk. Math.Fys. 21 (1944), 1-80.

[40] Pérez-Chavela E., Santoprete, M., Convex four-body central configurations with some equal masses, Arch. Rational Mech. Anal. 185 (2007), 481-494. 
[41] Piña, E., Lonngi, P., Central configuration for the planar Newtonian four-body problem, Celest. Mech. Dyn. Astron. 108 (2010), 73-93.

[42] Saari, D.G., On the role and properties of central configurations, Celestial Mech., 21 (1980), 9-20.

[43] Schmidt, D.S., Central configurations in $\mathbb{R}^{2}$ and $\mathbb{R}^{3}$, Contemporary Math. 81(1980), 59-76.

[44] Simó, C., Relative equilibrium solutions in the four-body problem, Cel. Mechanics 18 (1978), 165-184.

[45] Smale, S., Topology and mechanics II. The planar n-body problem, Invent.Math. 11 (1970), 45-64.

[46] Usiskin,Z., Griffin,J., Witonsky,D., Willmore,E., the classification of quadrilaterals: A study of definition, Research in mathematics education, IAP, (2008), 63-65.

[47] Wintner, A., The Analytical Foundations of Celestial Mechanics, Princeton Math. Series 5, Princeton University Press, Princeton, NJ, 1941.

[48] Xia, Z., Central configurations with many small masses, J. Differential Equations 91 (1991), 168-179.

[49] Xia, Z., Convex central configurations for the n-body problem, J. Differential Equations 200 (2004), 185-190.

[50] Xie, Z., Isosceles trapezoid central configurations of the Newtonian four-body problem, Proc. R. Soc. Edinb., Sect. A, Math. 142 (2012), 665-672.

${ }^{1}$ Departament de Matemàtiques, Universitat Autònoma de Barcelona, 08193 Bellaterra, Barcelona, Catalonia, Spain

E-mail address: jllibre@mat.uab.cat

2 School of Mathematics and Statistics, Southwest University, 400715, Chongqing, China

E-mail address: yuanpengfei@swu.edu.cn 